\title{
Passive laser irradiation as a tool for optical catalysis
}

Forbes, Kayn, Andrews, David

Kayn A. Forbes, David L. Andrews, "Passive laser irradiation as a tool for optical catalysis," Proc. SPIE 11345, Nanophotonics VIII, 113450G (1 April 2020); doi: $10.1117 / 12.2555047$

SPIE. Event: SPIE Photonics Europe, 2020, Online Only, France 


\title{
Passive laser irradiation as a tool for optical catalysis
}

\author{
Kayn A. Forbes and David L. Andrews \\ School of Chemistry, University of East Anglia, Norwich NR4 7TJ, United Kingdom \\ k.forbes@uea.ac.uk d.1.andrews@uea.ac.uk
}

\begin{abstract}
The mechanisms of absorption, emission, and scattering of photons form the foundations of optical interactions between light and matter. In the vast majority of such interactions there is a significant interplay and energy exchange between the radiation field and the material components. In absorption for example, modes of the field are depopulated by photons whose energy is at resonance with a molecular transition producing excited material states. In all such optical phenomena, the initial state of the radiation field differs in mode occupation to its final state. However, certain optical processes can involve off-resonance laser beams that are unchanged on interaction with the material: the output light, after interaction, is identical to the laser input. Such off-resonance interactions include forward Rayleigh scattering, responsible for the wellknown gradient force in optical trapping, and the laser-induced intermolecular interaction commonly termed optical binding; in both processes, an intense beam delivers its effect without suffering change. It is possible for beams detuned from resonance to provide not only techniques for optomechanical and optical manipulation, but also to passively influence other important and functional interactions such as absorption from a resonant beam, or energy transfer. Such effects can be grouped under the banner of 'optical catalysis', since they can significantly influence resonant processes. Furthermore, off-resonance photonics affords a potential to impact on chemical interactions, as in the passive modification of rotational constants and phase transitions. To date, apart from optical manipulation, the potential applicability of passive photonics, particularly in the realm of chemical physics and materials science, has received little attention. Here we open up this field, highlighting the distinct and novel role that off-resonance laser beams and the ensuing photonics can play.
\end{abstract}

Keywords: off-resonance, laser detuning, optical catalysis, optical binding, optical trapping, phase transitions, optical manipulation, passive photonics

\section{INTRODUCTION}

The quantum structure of atoms and molecules leads to discrete energy levels which can only be excited by light of an appropriate frequency to match the energy gap of any particular transition: the quantum aspects of both materials and light represent a principle that underpins absorption and emission spectroscopy ${ }^{1,2}$. It is of course possible to subject transparent materials to off-resonant laser beams and observe optical-matter interactions - Raman scattering, for example. In a sense, therefore, it is helpful to recognize one common feature: an input laser beam undergoes change as a result of its interaction with the matter. This is true, for example, for both Raman scattering and non-forward Rayleigh scattering. From a photon perspective, the occupancy of an input mode $(\boldsymbol{k}, \eta)$ of wave vector $\boldsymbol{k}$ and polarization $\eta$ is changed in the course of both these scattering processes. It is, however, possible for an off-resonant laser to emerge from material with its modal composition unaltered, and yet deliver a physical effect. The most fundamental application of a detuned laser of this type is forward Rayleigh scattering. In forward Rayleigh scattering the input and output photon are in an identical mode, and no energy or momentum can be imparted to, or taken from, the material. Nonetheless the throughput electromagnetic 
fields can perturb the electronic energy levels to the extent that position-dependent dynamic Stark shifts are introduced, resulting in a light-induced potential energy field. The gradient force in optical trapping thus arises, as transparent particles are attracted to a high-intensity region of light ${ }^{3}$.

Forward Rayleigh scattering mediated by two or more particles leads to optical binding forces: a mutual interparticle interaction energy. The off-resonance processes of trapping and binding are extremely well utilized in the field of optical nanomanipulation ${ }^{4}$. In this paper we study distinctly different applications: the passive effects of an off-resonance or detuned laser grouped under the banner of 'optical catalysis'. Firstly we survey the role of an off-resonant beam on the resonant processes of absorption, emission and energy transfer. Secondly we study the application of an off-resonant beam, through binding and trapping forces, in areas beyond optical manipulation. We utilize the theory of quantum electrodynamics ${ }^{5}$ to describe the interactions, giving an indicative explicit calculation in the case of laser-modified absorption, and our work fully embraces the concept and physics of the photon.

It is important to make clear at this early stage that our definition of optical catalysis is distinctly different to that of photocatalysis: the former rests upon the realization that off-resonant laser beams can influence transparent molecules without suffering change in their modal composition (i.e. the light is the catalyst which suffers no degradation); the latter is the well-known acceleration of a photoreaction due to the absorption of light and thus the transfer of energy from the laser to the material. However, as we shall show, there are indeed emerging applications of the optics-based definition that do now relate to the field of chemistry, in which the scientific term 'catalysis' was first introduced by Berzelius ${ }^{6}$.

\section{OPTICAL CATALYSIS OF LIGHT-MATTER PROCESSES}

The simplest optical process based on a resonant interaction between light and matter is, of course, single-photon absorption from a resonant optical input. Consider such a process taking place in the presence of a secondary, off-resonant beam. For a molecule $\xi$ in the presence of $n$ photons of the resonant mode $(\boldsymbol{k}, \eta)$ and $n^{\prime}$ photons of the off-resonant mode $\left(\boldsymbol{k}^{\prime}, \eta^{\prime}\right)$ in an arbitrary quantization volume $V$, the initial and final states of the system, $|i\rangle$ and $|f\rangle$ respectively, explicitly represent the product matter-radiation states $\left|E_{0}(\xi)\right\rangle\left|n(\boldsymbol{k}, \eta) ; n^{\prime}\left(\boldsymbol{k}^{\prime}, \eta^{\prime}\right)\right\rangle$ and $\left|E_{\alpha}(\xi)\right\rangle\left|(n-1)(\boldsymbol{k}, \eta) ; n^{\prime}\left(\boldsymbol{k}^{\prime}, \eta^{\prime}\right)\right\rangle$. In order to calculate the quantum amplitudes $M_{f i}$ for resonant absorption by this system, in the presence of the off-resonant beam, we deploy the Feynman time-ordered diagrams in Figure 1, engaging both first- and third-order perturbation theory:

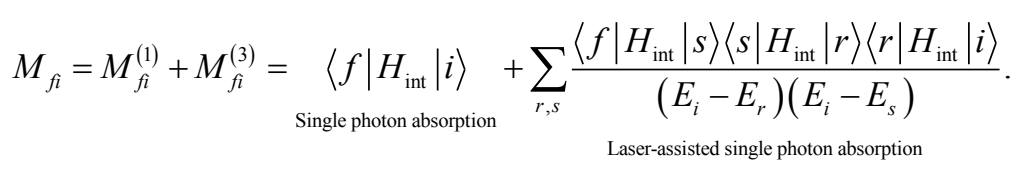

In (1), $H_{\text {int }}$ is the Power-Zienau-Woolley (PZW) quantum electrodynamical interaction Hamiltonian operator responsible for state transitions: in this analysis we restrict ourselves to the electric-dipole approximation, most simply expressed as $H_{\text {int }}=-\boldsymbol{\mu} \cdot \boldsymbol{e}^{\perp}$ where $\boldsymbol{\mu}$ is the electric-dipole operator and $\boldsymbol{e}^{\perp}$ is the transverse electric field operator. The term arising from third-order perturbation provides the leading contribution that can engage off-resonant light ${ }^{7}$. 

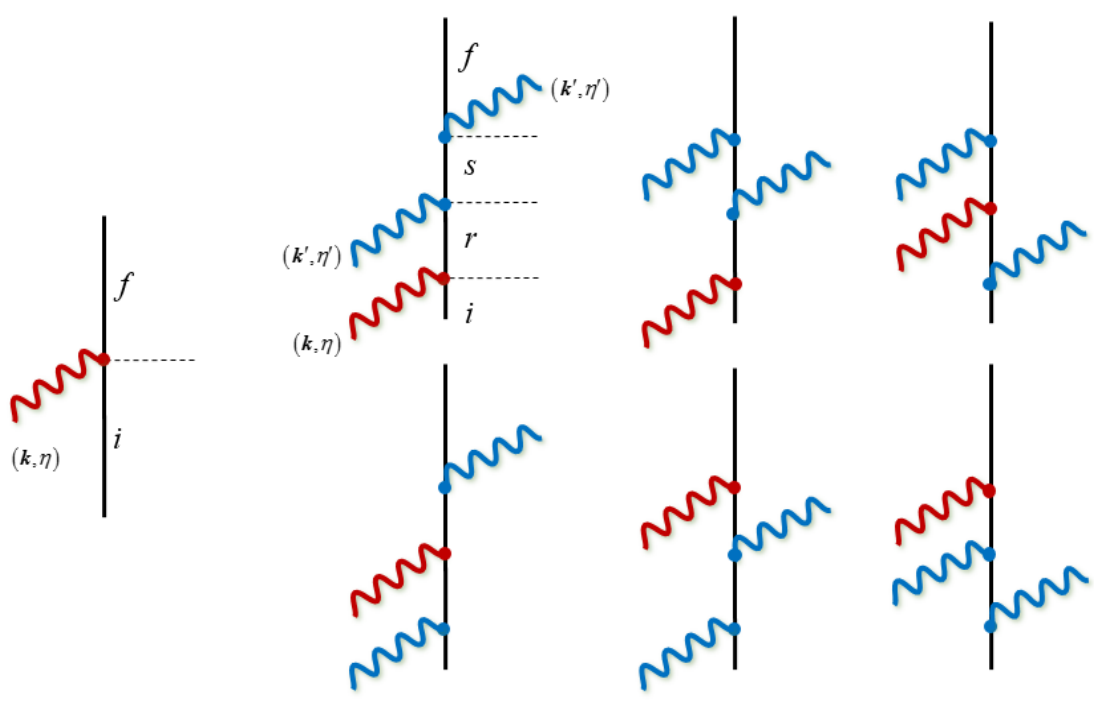

Figure 1: Time-ordered Feynman diagrams representing single-photon absorption and laser-modified single-photon absorption. Red wavy lines represent the resonant photon of $\operatorname{mode}(\boldsymbol{k}, \eta)$ that is absorbed; blue wavy lines represent the forward Rayleigh scattered photons of mode $\left(\boldsymbol{k}^{\prime}, \eta^{\prime}\right)$.

Carrying out the operations in (1), to couple the given initial and final states of the system, using the standard mode expansions for the electric field operator, leads to the single-photon absorption quantum amplitude (from the first term) and a laser-assisted single-photon absorption amplitudes (second term). Their sum, for a system of $N$ molecules labeled $\xi$, at positions $\boldsymbol{R}_{\xi}$, emerges in the following form, in which $\boldsymbol{e}^{(\prime)}$ is the polarization vector for the photons of mode $\boldsymbol{k}^{(\prime)}$ per quantization volume $V$, and an overbar represents complex conjugation, to allow for circular or elliptical polarizations:

$M_{f i}=M_{f i}^{(1)}+M_{f i}^{(3)}=-i\left(\frac{n c k}{2 \varepsilon_{0} V}\right)^{\frac{1}{2}} e_{i} \sum_{\xi}^{N} \mu_{i}^{\alpha 0}(\xi) \mathrm{e}^{i \boldsymbol{k} \cdot \boldsymbol{R}_{\xi}}-i \sqrt{n k} n^{\prime} k^{\prime}\left(\frac{c}{2 \varepsilon_{0} V}\right)^{\frac{3}{2}} e_{i} e_{j}^{\prime} \overline{e_{k}^{\prime}} \sum_{\xi}^{N} \beta_{i j k}^{\alpha 0}(\xi) \mathrm{e}^{i \boldsymbol{k} \cdot \boldsymbol{R}_{\xi}} \mathrm{e}^{i \boldsymbol{k}^{\prime} \cdot \boldsymbol{R}_{\xi}} \mathrm{e}^{-i \boldsymbol{k}^{\prime} \cdot \boldsymbol{R}_{\xi}}$

Here, also, $\boldsymbol{\mu}^{\alpha 0}$ and $\boldsymbol{\beta}^{\alpha 0}$ respectively represent the transition dipole moment and transition hyperpolarizability, and the repeated subscript indices, denoting Cartesian components, are subject to the convention of summation. Full details are given in ref. ${ }^{7}$ We may now proceed to full calculation of the rate, $\Gamma$, for the absorption via the Fermi Rule, $\Gamma=2 \pi^{-1} \rho\left|M_{f i}^{(1)}+M_{f i}^{(3)}\right|^{2}$. The terms with the largest contribution to absorption are $M_{f i}^{(1)} \bar{M}_{f i}^{(1)}, M_{f i}^{(1)} \bar{M}_{f i}^{(3)}, \bar{M}_{f i}^{(1)} M_{f i}^{(3)}$ : recasting these in terms of the two beam intensities, $I$ and $I^{(t)}$, gives:

$\Gamma \approx \frac{N \pi \rho I}{c \varepsilon_{0}} e_{i} \bar{e}_{l}\left[\mu_{i}^{\alpha 0}(\xi) \bar{\mu}_{l}^{\alpha 0}(\xi)+\left(\frac{I^{\prime}}{c \varepsilon_{0}}\right) e_{j}^{\prime} \bar{e}_{k}^{\prime}\left(\mu_{i}^{\alpha 0}(\xi) \beta_{l k j}^{\alpha 0}(\xi)+\bar{\mu}_{l}^{\alpha 0}(\xi) \beta_{i j k}^{\alpha 0}(\xi)\right)\right]$

The above analysis, illustrating derivation of the effect of the passive beam on single-photon absorption, has been extended in other previous work to apply to laser-modified two-photon absorption ${ }^{7,8}$. An alternative route to the same result is to determine corrections to the material wavefunctions resulting from the passive beam - as a second-order (dynamic) Stark effect - and then to use the corrected states as a basis for the normal calculation of single-photon absorption. 
Using a detuned laser to assist or modify the efficiency of photon absorption is just one of a plethora of optical catalysis mechanisms ${ }^{9}$. Further examples have been explored in considering the two major relaxation routes of an electronically excited molecule: fluorescence and resonant energy transfer (RET). Application of off-resonance light to both these processes lead to increased control through laser-modified fluorescence and laser-assisted resonance energy transfer $(\text { LARET })^{10-15}$. In laser-assisted fluorescent emission, the forward Rayleigh scattering of the detuned laser leads to either an enhanced or suppressed rate of fluorescent emission, in an analogous fashion to the effect on absorption. It is important to note, however, that this mechanism is distinctly different from stimulated emission, which involves the presence of radiation with a frequency which must match that of the fluorescence. The influence of the detuned laser on fluorescence is measurable through modifications to the fluorescence polarization anisotropy, fluorophore decay lifetimes, and altered near-field distributions of fluorescence.

\section{OPTICAL CATALYSIS OF LIGHT-MATTER PHENOMENA}

The light-matter interactions described in Section 2 are strictly termed optical processes, as their matrix elements are used to calculate a rate (via the Fermi rule); here a distinction can be made from optical phenomena in which the calculated matrix element represents a potential energy rather than a rate ${ }^{16}$. The crux of whether any given light-matter interaction falls into one or other of these categories is simple enough: in phenomena that yield potential energies and forces (this force being calculated through the negative gradient of the potential energy) the initial and final states of the radiation field are exactly identical; in all other cases the interaction is a process, and the matrix element delivers a rate.

The two differing origins of molecular motion that arise through laser irradiation reflect the same distinction. In absorption or scattering processes it is the delivery of momentum that produces the motion of particles, whereas in a potential energy or force it is an associated positional or angle-dependence which, as a secondary effect, yields particle motion. Putting inter-particle interactions aside for a moment, the most well-known of the latter optical forces is the gradient force, which constitutes part of the total optical tweezer effect ${ }^{17}$. At the photon level the gradient force occurs through a forward Rayleigh scattering mechanism, which can again be viewed as a dynamic Stark shift. Simple quantum electrodynamical methods yield the potential energy, for an isotropic molecule of frequency-dependent polarizability $\alpha$, as;

$$
\left\langle\Delta E_{\text {grad }}(\boldsymbol{r})\right\rangle=-\frac{I(\boldsymbol{r})}{6 \varepsilon_{0} c} \alpha(\omega)
$$

That is to say, molecules which scatter light in the forward direction, where the material suffers no change of electronic state, experience a potential energy which is dependent on position $\boldsymbol{r}$ in the beam. The associated force, readily calculated by taking the gradient of (4), usually serves to direct particles to the position of highest intensity in the beam. Evidently, on the throughput of a detuned laser beam, we observe a physical effect even though both the radiation and material suffers no overall change during the interaction.

Laser-induced modifications to the well-known London or Casimir-Polder dispersion forces are another manifestation of optical force. Fundamental electrodynamics provides a mechanism for forward Rayleigh scattering between two or more particles, mediated by a virtual photon, the intermolecular interaction leading to a pair energy that is once again dependent on the intensity of the input laser beam ${ }^{18,19}$. This is known as optical binding ${ }^{20}$. This laser-induced force between particles is completely distinct (though complementary in the field of optical manipulation) from the gradient force in optical trapping which acts on single particles. The extent and significance of optical binding are dependent on numerous parameters, such as the interparticle separation, input polarization, laser intensity, and polarizability of the particles. This latter dependence on material composition is indirectly size-dependent for individual molecules; in larger dielectric particles where linear susceptibility is the corresponding measure there is often a more explicit size-dependence, as for example in Clausius-Mosotti and Mie models. This is the main reason why the majority of experimental studies on optical binding so far have been in the size range of microscopic particles. However, with improving experimental methodologies, 
including highly stable trapping arrangements and the use of plasmonic particles, observation of optical binding at the nanoscale has evolved significantly in the last half-decade, even extending to dielectric nanowires ${ }^{21}$; the reader is referred to recent overviews for more general detail $\mathrm{s}^{20,22}$.

In the field of optical manipulation, small numbers of particles are trapped and studied in experimentally confined geometrical configurations, and so averaging over values of the interparticle displacement vector is not usually necessary. The general form of optical binding between two molecules A and B in this scenario is;

$\Delta E=\left(\frac{I}{\varepsilon_{0} c}\right) \alpha_{\mathrm{A}}(\omega) \alpha_{\mathrm{B}}(\omega) \bar{e}_{i}^{(\eta)} e_{j}^{(\eta)} \operatorname{Re} V_{i j}(k, \boldsymbol{R}) \cos (\boldsymbol{k} \cdot \boldsymbol{R})$

where the second rank tensor $\boldsymbol{V}$ signifies the retarded resonance dipole-dipole interaction ${ }^{23}$. Numerous specific results can be derived using this powerful equation, first derived by Thirunamachandran using QED methods, as a starting point ${ }^{18}$.

\section{ROLE OF OPTICAL CATALYSIS IN CHEMICAL SCIENCES}

The utilization and application of the gradient force and optical binding interactions have largely been in the field of optical manipulation. Individual particles can be trapped and manipulated using optical tweezers, whilst so-called 'optical matter ${ }^{24}$ is largely due to a combination of optical trapping and binding forces in multi-particle ensembles. In both of these cases, the very weak forces acting on individual particles are constantly undermined by Brownian motion and other disruptive interactions. A very under-appreciated fact until now is that both these mechanisms still act out over different scales - part of the significance being the highly intricate form and span of potential energy landscapes generated by the optical binding ${ }^{25}$. (As noted in many publications, the term is unfortunate since it quite wrongly implies a monotonic, attractive force.)

At the intramolecular atomic scale, for example, the optical binding interaction can exert an influence in van der Waals molecules $(\mathrm{VWM})^{26}$. These molecules are weakly bound, usually dimeric molecular structures, which are readily identifiable by high resolution microwave spectroscopy. The $(\mathrm{HCN})_{2}$ dimer is a well-known A-B type (Figure 2) whose intermolecular bond is modelled by the Stockmayer potential:

$U\left(\boldsymbol{R}, \boldsymbol{\mu}^{\mathrm{A}}, \boldsymbol{\mu}^{\mathrm{B}}\right)=4 \varepsilon\left[(\sigma / R)^{12}-(\sigma / R)^{6}\right]+\boldsymbol{\mu}^{\mathrm{A}} \cdot \boldsymbol{\mu}^{\mathrm{B}} / 4 \pi \varepsilon_{0} R^{3}-3\left(\boldsymbol{\mu}^{\mathrm{A}} \cdot \boldsymbol{R}\right)\left(\boldsymbol{\mu}^{\mathrm{B}} \cdot \boldsymbol{R}\right) / 4 \pi \varepsilon_{0} R^{5}$

where $\boldsymbol{R}$ is the intermolecular separation vector, $\sigma$ is the Lennard-Jones parameter, $\varepsilon$ is the well depth and the $\mu$ terms are the dipole moments. Inclusion of the optical binding interaction leads to an effective potential $U(\boldsymbol{R})+\Delta E(\boldsymbol{R})$. The modification of the potential energy surface due to the off-resonant laser changes the equilibrium bond length in a contraction or expansion, dependent on whether the frequency-dependent optical binding force is positive or negative. 


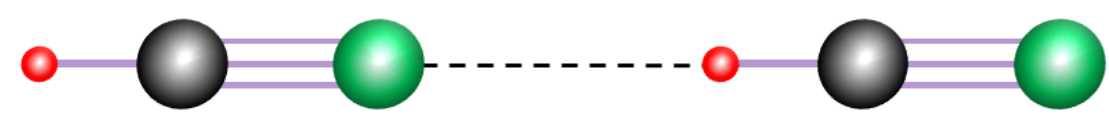

Figure 2: $(\mathrm{HCN})_{2}$ dimer. Small red balls represent hydrogen; green are nitrogen; and black are carbon. The dashed lined represents the intermolecular hydrogen bonding between hydrogen and nitrogen.

As a result of this interaction, therefore, a new equilibrium bond length is established for VMW molecules within the laser beam, modifying their moments of inertia and hence the rotational constant of the dimer. It has been established that for a relatively modest irradiance of $10^{12} \mathrm{~W} \mathrm{~cm}^{-2}$ the dimer will extend by $1.72 \mathrm{pm}$, which yields a new rotational constant $0.0579 \mathrm{~cm}^{-1}$. This value of the rotational constant of the dimer when under passive irradiation is different by $1 \%$ from the rotational constant without the influence of optical binding; a value that is experimentally significant and well above the very low bounds of error in microwave spectroscopic measurements.

At the opposite end of the scale in chemical application are the effects of imposing an optically conferred potential energy landscape on bulk collections of molecules and particles. Even if such systems do not afford the same control of nanomanipulation physics, a large sample of molecules will still feel the potential energy lowering effect of the gradient and binding forces. Depending on the specific physical or chemical interaction under study, the presence of the passive laser responsible for these interactions can exert significant influence. For example, passive radiation may play a significant role is in bulk interactions and phase transitions. In particular, the optical trapping and binding interactions between molecules should act as a degree of control in certain scenarios, such as in phase transitions, where laser-tweezing is known to operate ${ }^{27}$. It is clear that in a two-component system (for example) consisting of many $\mathrm{A}$ and $\mathrm{B}$ molecules, then in comparison to other laser-induced forces that will be equal in the reactant and product states, the optical binding interaction between A and B could play a pivotal, equilibrium-tipping role in determining whether or not the final state is kinetically favourable.

Beyond these specifically physical processes, more in connection with chemical reaction, it is also interesting to note a connection with non-resonant photocatalysis. Alternatively called dynamic Stark control ${ }^{28}$, this recently introduced method uses acutely timed off-resonant pulses of light to act as a catalyst in intramolecular bond-breaking reactions. Thus far, this type of photonic catalysis has successfully been used in photochemical dissociation reactions of diatomics ${ }^{29}$, polyatomics ${ }^{30,31}$, and phenol $^{32}$. As noted earlier, the photonic mechanism for the dynamic Stark shift harnessed in these reactions is simply forward Rayleigh scattering - which, as we have seen, is the same passive mechanism behind the gradient force in optical trapping. The optical engineering of dynamic Stark control precludes a plethora of competing chemical reactions that might otherwise compete, if absorption or ionization were to occur. In a QED photonic description, dynamic Stark control of this kind comprises conventional, single-photon absorption, followed by the forward-Rayleigh scattering process that shifts the energy barrier in a stepwise mechanism.

\section{CONCLUSION}

It is clearly evident that a detuned, non-resonant laser can exhibit measurable interactions with matter. Importantly, the mechanisms whose theory has been discussed in Section 2 lead to a greatly expanded space in experimentally controllable parameters. The effect of the detuned laser beam in the mechanisms detailed in Section 3 and 4 is responsible for distinctive optical phenomena; the specific cases of the gradient force and optical binding are very well known and constitute the field of optical manipulation. However, we have highlighted here the potential for the optical binding interaction between particles to serve a highly novel role in bulk materials, which are the study of chemical physics and physical chemistry. We have specifically highlighted the role of bimolecular dynamic Stark shifts on phase separation and microwave rotational spectroscopy, as well highlighting the photonic mechanism behind non-resonant dynamic Stark control of photocatalysis. This opens up a distinct field in chemistry where resonant light in photocatalytic processes are already well known, but the benefits of off-resonant light should begin to bear additional gains. 


\section{ACKNOWLEDGEMENT}

K.A.F gratefully acknowledges the Leverhulme Trust for funding through a Leverhulme Early Career Fellowship, award no. ECF-2019-398.

\section{REFERENCES}

[1] Hollas, J. M., [Modern Spectroscopy], John Wiley \& Sons, Chichester, UK (2004).

[2] Demtröder, W., [Atoms, Molecules and Photons], Springer, New York (2010).

[3] Andrews, D. L. and Bradshaw, D. S., [Optical Nanomanipulation], Morgan \& Claypool Publishers, California (2017).

[4] Bradac, C., "Nanoscale Optical Trapping: A Review,” Adv. Opt. Mater., 1800005 (2018).

[5] Craig, D. P. and Thirunamachandran, T., [Molecular Quantum Electrodynamics: An Introduction to RadiationMolecule Interactions], Courier Corporation, New York (1998).

[6] Berzelius, J. J., "Quelques Idées sur une nouvelle Force agissant dans les Combinaisons des Corps Organiques," Ann Chim 61, 146-151 (1836).

[7] Bradshaw, D. S. and Andrews, D. L., "Laser-modified one-and two-photon absorption: Expanding the scope of optical nonlinearity,” Phys. Rev. A 88(3), 033807 (2013).

[8] Ford, J. S. and Andrews, D. L., "One-and two-photon absorption in solution: The effects of a passive auxiliary beam,” J. Chem. Phys. 141(3), 034504 (2014).

[9] Bradshaw, D. S., Forbes, K. A. and Andrews, D. L., "Off-resonance control and all-optical switching: expanded dimensions in nonlinear optics," Appl. Sci. 9(20), 4252 (2019).

[10] Allcock, P., Jenkins, R. D. and Andrews, D. L., "Laser-assisted resonance-energy transfer," Phys. Rev. A 61(2), $023812(2000)$.

[11] Bradshaw, D. S. and Andrews, D. L., "Optically controlled resonance energy transfer: Mechanism and configuration for all-optical switching,” J. Chem. Phys. 128(14), 144506 (2008).

[12] Bradshaw, D. S. and Andrews, D. L., “All-optical control of molecular fluorescence," Phys. Rev. A 81(1), 013424 (2010).

[13] Andrews, D. L. and Bradshaw, D. S., “Off-resonant activation of optical emission,” Opt. Commun. 283(21), 43654367 (2010).

[14] Leeder, J. M., Bradshaw, D. S. and Andrews, D. L., "Laser-controlled fluorescence in two-level systems," J. Phys. Chem. B 115(18), 5227-5233 (2011).

[15] Weeraddana, D., Premaratne, M., Gunapala, S. D. and Andrews, D. L., "Quantum electrodynamical theory of highefficiency excitation energy transfer in laser-driven nanostructure systems," Phys. Rev. B 94(8), 085133 (2016).

[16] Bradshaw, D. S. and Andrews, D. L., "Manipulating particles with light: Radiation and gradient forces," Eur. J. Phys. 38(3), 034008 (2017).

[17] Bradac, C., “Nanoscale optical trapping: A review,” Adv. Opt. Mater. 6(12), 1800005 (2018). 
[18] Thirunamachandran, T., "Intermolecular interactions in the presence of an intense radiation field," Mol. Phys. 40(2), 393-399 (1980).

[19] Salam, A., [Molecular Quantum Electrodynamics: Long-range Intermolecular Interactions], John Wiley \& Sons, New Jersey (2010).

[20] Dholakia, K. and Zemánek, P., “Colloquium: Gripped by light: Optical binding,” Rev. Mod. Phys. 82(2), 1767 (2010).

[21] Donato, M. G., Brzobohatý, O., Simpson, S. H., Irrera, A., Leonardi, A. A., Lo Faro, M. J., Svak, V., Marago, O. M. and Zemánek, P., "Optical Trapping, Optical Binding, and Rotational Dynamics of Silicon Nanowires in Counter-Propagating Beams," Nano Lett. 19(1), 342-352 (2018).

[22] Forbes, K. A., Bradshaw, D. S. and Andrews, D. L., “Optical binding of nanoparticles,” Nanophotonics 9(1), 1-17 (2019).

[23] Daniels, G. J., Jenkins, R. D., Bradshaw, D. S. and Andrews, D. L., "Resonance energy transfer: The unified theory revisited," J. Chem. Phys. 119(4), 2264-2274 (2003).

[24] Burns, M. M., Fournier, J.-M. and Golovchenko, J. A., "Optical matter: crystallization and binding in intense optical fields," Science 249(4970), 749-754 (1990).

[25] Rodríguez, J., Romero, L. C. D. and Andrews, D. L., "Optical binding in nanoparticle assembly: Potential energy landscapes,” Phys. Rev. A 78(4), 043805 (2008).

[26] Andrews, D. L., Crisp, R. G. and Bradshaw, D. S., "Optically induced inter-particle forces: from the bonding of dimers to optical electrostriction in molecular solids," J. Phys. B At. Mol. Opt. Phys. 39(15), S637 (2006).

[27] Walton, F. and Wynne, K., "Control over phase separation and nucleation using a laser-tweezing potential," Nat. Chem. 10(5), 506-510 (2018).

[28] Sussman, B. J., Townsend, D., Ivanov, M. Y. and Stolow, A., "Dynamic stark control of photochemical processes," Science 314(5797), 278-281 (2006).

[29] Hilsabeck, K. I., Meiser, J. L., Sneha, M., Balakrishnan, N. and Zare, R. N., "Photon catalysis of deuterium iodide photodissociation," Phys. Chem. Chem. Phys. 21(26), 14195-14204 (2019).

[30] Corrales, M. E., González-Vázquez, J., Balerdi, G., Solá, I. R., De Nalda, R. and Bañares, L., "Control of ultrafast molecular photodissociation by laser-field-induced potentials," Nat. Chem. 6(9), 785-790 (2014).

[31] Corrales, M. E., de Nalda, R. and Bañares, L., "Strong laser field control of fragment spatial distributions from a photodissociation reaction," Nat. Commun. 8(1), 1-7 (2017).

[32] Hilsabeck, K. I., Meiser, J. L., Sneha, M., Harrison, J. A. and Zare, R. N., "Nonresonant Photons Catalyze Photodissociation of Phenol," J. Am. Chem. Soc. 141(2), 1067-1073 (2019). 